\title{
Ethnic differences in disease presentation of uterine cancer in New Zealand women
}

\author{
Ridvan Tupai Firestone, ${ }^{1}$ Lis Ellison-Loschmann, ${ }^{2}$ Andrew N Shelling, ${ }^{3}$ Alec Ekeroma, ${ }^{4}$ \\ Bettina A Ikenasio-Thorpe, ${ }^{5}$ Neil Pearce, ${ }^{6}$ Mona Jeffreys ${ }^{7}$
}

\author{
'Research Fellow, Centre for \\ Public Health Research, Massey \\ University, Wellington, New \\ Zealand \\ ${ }^{2}$ Research Fellow, Centre for \\ Public Health Research, Massey \\ University, Wellington, New \\ Zealand \\ ${ }^{3}$ Associate Professor, Department \\ of Obstetrics and Gynecology, \\ Faculty of Medical and Health \\ Sciences, University of Auckland, \\ Auckland, New Zealand \\ ${ }^{4}$ Head of Department, Pacific \\ Women's Health Research and \\ Development Unit, Department \\ of Obstetrics and Gynaecology, \\ University of Auckland, \\ Auckland, New Zealand \\ ${ }^{5}$ Lead Researcher, Pacific Health \\ Research and Development \\ Foundation/Pacific Women's \\ Health Research and \\ Development Unit, Auckland, \\ New Zealand \\ ${ }^{6}$ Professor, Centre for Public \\ Health Research, Massey \\ University, Wellington, New \\ Zealand \\ ${ }^{7}$ Senior Lecturer, Department of \\ Social Medicine, University of \\ Bristol, Bristol, UK
}

\section{Correspondence to}

Dr Ridvan Tupai Firestone, Centre for Public Health Research, Massey University, Private Box 756, Wellington, New Zealand; R.T.Firestone@massey.ac.nz

Received 28 March 2011 Accepted 16 November 2011

Published Online First

12 January 2012

\begin{abstract}
Objectives Little is known about the ethnic differences in disease presentation of uterine cancer in New Zealand women. The objectives of this study were two-fold: (1) to estimate the incidence and mortality of uterine cancer among women in New Zealand and (2) to

Key message points

- There is substantial variation in the incidence of uterine cancer by ethnicity, with the highest incidence and late stage of disease noted primarily in Pacific and Māori women.

- There also appears to be a socioeconomic gradient in the risk of uterine cancer with higher incidence in more deprived groups, but the reasons for this are unclear.
\end{abstract} examine the association of ethnicity and socioeconomic status with tumour stage and grade, at presentation of uterine cancer. Methods Retrospective survey of cancer cases identified from the New Zealand Cancer Registry. The authors analysed all 3203 uterine cancer cases registered with the New Zealand Cancer Registry during the period 1 January 1997 to 31 December 2006. Ethnic groups were defined based on the self-identified ethnicity recorded on the cancer registry: Māori, Pacific and non-Māori non-Pacific women. Socioeconomic status was categorised as quintiles of the New Zealand Deprivation Index 2006. The mortality to incidence ratio was used as a measure of prognosis. Logistic regression was used to estimate age, ethnic and deprivation adjusted odds ratios (ORs) and $95 \%$ confidence intervals (Cls).

Results Pacific and Māori women have higher incidence (32.4 and 17.7 per 100000 women, respectively) and mortality rates of uterine cancer (12.1 and 7.4 per 100000 women, respectively). Women in the most deprived areas are more likely to present with an advanced stage of uterine cancer (OR 1.64, 95\% Cl 1.09-2.48). Māori and Pacific women are less likely to present with well-differentiated tumours (OR 0.69, 95\% Cl 0.52-0.92 and OR $0.72,95 \% \mathrm{Cl} 0.52-0.99$, respectively).

Conclusions Māori and Pacific women, and those from lower socioeconomic areas, are more likely to present with advanced uterine cancer.

\section{Introduction}

The majority of uterine cancers are derived from the endometrial tissue within the inner layer of the uterus; hence they are referred to as endometrial cancers. These are the most frequently diagnosed genital cancers, accounting for $4 \%$ of all female cancers worldwide, and they are the fourth most common cancer in women from developed countries. ${ }^{1} 2$ In New Zealand in 2005, the age-standardised rate for cancer of the uterus was higher (13.8 per 100 000) than those for cancer of the ovary (10.5 per 100000$)$ and of the cervix (6.2 per 100000$).{ }^{3}$ Age-standardised incidence rates are projected to remain stable over a 15-year period (1997-2012), ${ }^{4}$ a trend that was also projected in the USA. ${ }^{5}$ In New Zealand, mortalities from uterine cancer have declined since the 1970s, but there was a slight increase reported in the late 1990s. ${ }^{4}$ Currently, the age-standardised mortality rate from cancer of the uterus $(2.6$ per 100000$)$ is intermediary, compared to cancers of the ovary (6.1 per $100000)$ and cervix (1.9 per 100000$).^{3}$ Uterine cancer has a relatively good prognosis (death to registration ratio, 0.21 ). ${ }^{3}$

Few studies have examined the association between socioeconomic status and uterine cancer. It has been reported that the incidence of uterine cancer was more common in women of higher socioeconomic status, in Westernised countries. ${ }^{5} 6$ However, in New Zealand, the incidence and mortality from uterine cancer is higher in women from lower socioeconomic incidence of, and mortality from, uterine cancer in New Zealand women. It also assesses the contributions of ethnicity and socioeconomic status to tumour stage and grade, at presentation of uterine cancer. groups. ${ }^{3-9}$ This article reports the recent 
Table 1 Distributions of uterine cancer characteristics, by ethnicity

\begin{tabular}{|c|c|c|c|c|c|c|c|c|}
\hline \multirow[b]{2}{*}{ Age group (years) } & \multicolumn{2}{|c|}{$\mathrm{nMnP}(n=2564)$} & \multicolumn{2}{|c|}{ Māori $(n=366)$} & \multicolumn{2}{|c|}{ Pacific $(n=273)$} & \multicolumn{2}{|c|}{ All women $(n=3203)$} \\
\hline & $n$ & $\%$ & $n$ & $\%$ & $n$ & $\%$ & $n$ & $\%$ \\
\hline $15-19$ & 1 & 0.04 & - & - & 1 & 0.37 & 2 & 0.06 \\
\hline $20-24$ & 2 & 0.08 & - & - & - & - & 2 & 0.06 \\
\hline $25-29$ & 2 & 0.08 & 2 & 0.54 & 2 & 0.73 & 6 & 0.19 \\
\hline $30-34$ & 17 & 0.66 & 8 & 2.18 & 9 & 3.29 & 34 & 1.06 \\
\hline $35-39$ & 28 & 1.09 & 9 & 2.46 & 14 & 5.13 & 51 & 1.59 \\
\hline $40-44$ & 62 & 2.42 & 31 & 8.47 & 21 & 7.69 & 114 & 3.56 \\
\hline 45-49 & 130 & 5.07 & 46 & 12.57 & 24 & 8.79 & 200 & 6.24 \\
\hline $50-54$ & 249 & 9.71 & 66 & 18.03 & 32 & 11.72 & 347 & 10.83 \\
\hline $55-59$ & 395 & 15.41 & 61 & 16.67 & 32 & 11.72 & 488 & 15.23 \\
\hline $60-64$ & 362 & 14.12 & 46 & 12.57 & 48 & 17.58 & 456 & 14.24 \\
\hline $65-69$ & 348 & 13.57 & 47 & 12.84 & 42 & 15.38 & 437 & 13.64 \\
\hline $70-74$ & 332 & 12.56 & 21 & 5.74 & 21 & 7.69 & 374 & 10.83 \\
\hline $75-79$ & 252 & 9.83 & 17 & 4.64 & 17 & 6.22 & 286 & 8.93 \\
\hline $80-84$ & 226 & 8.81 & 7 & 1.91 & 7 & 2.56 & 240 & 7.49 \\
\hline $\begin{array}{l}85+ \\
p<0.001\end{array}$ & 158 & 6.16 & 5 & 1.37 & 3 & 1.10 & 166 & 5.18 \\
\hline \multicolumn{9}{|l|}{ NZDep06* } \\
\hline $1-2$ & 441 & 17.2 & 15 & 4.1 & 10 & 3.7 & 466 & 14.5 \\
\hline $3-4$ & 454 & 17.7 & 31 & 8.5 & 18 & 6.6 & 503 & 15.7 \\
\hline $5-6$ & 523 & 20.4 & 38 & 10.4 & 40 & 14.6 & 601 & 18.8 \\
\hline $7-8$ & 691 & 26.9 & 81 & 22.1 & 53 & 19.4 & 825 & 25.8 \\
\hline $9-10$ & 446 & 17.4 & 201 & 54.9 & 147 & 53.9 & 794 & 24.8 \\
\hline $\begin{array}{l}\text { Missing } \\
p<0.001\end{array}$ & 9 & 0.4 & - & - & 5 & 1.8 & 14 & 0.4 \\
\hline \multicolumn{9}{|l|}{ Cell type } \\
\hline Endometroid & 2052 & 80.0 & 289 & 78.9 & 206 & 75.5 & 2547 & 79.5 \\
\hline Adenosquamous & 126 & 5.0 & 11 & 3.0 & 15 & 5.5 & 152 & 4.8 \\
\hline Clear cell & 21 & 0.8 & 5 & 1.4 & 2 & 0.7 & 28 & 0.9 \\
\hline Mucinous & 12 & 0.5 & 1 & 0.3 & 1 & 0.4 & 14 & 0.4 \\
\hline Squamous cell & 216 & 8.4 & 45 & 12.3 & 31 & 11.4 & 292 & 9.1 \\
\hline $\begin{array}{l}\text { Undifferentiated } \\
p=0.186\end{array}$ & 137 & 5.3 & 15 & 4.1 & 18 & 6.5 & 170 & 5.3 \\
\hline \multicolumn{9}{|l|}{ Stage } \\
\hline Local & 293 & 11.4 & 41 & 11.2 & 20 & 7.3 & 354 & 11.1 \\
\hline Regional & 1829 & 71.3 & 257 & 70.2 & 170 & 62.3 & 2256 & 70.4 \\
\hline Distant & 201 & 7.8 & 37 & 10.1 & 43 & 15.7 & 281 & 8.8 \\
\hline $\begin{array}{l}\text { Unknown } \\
p<0.001\end{array}$ & 241 & 9.4 & 31 & 8.5 & 40 & 14.7 & 312 & 9.7 \\
\hline \multicolumn{9}{|l|}{ Grade } \\
\hline Well differentiated & 866 & 33.8 & 123 & 33.6 & 93 & 34.1 & 1082 & 33.8 \\
\hline Moderately differentiated & 379 & 14.8 & 61 & 16.7 & 33 & 12.1 & 473 & 14.8 \\
\hline Poorly differentiated & 299 & 11.6 & 48 & 13.1 & 48 & 17.6 & 395 & 12.3 \\
\hline Undifferentiated & 7 & 0.3 & 3 & 0.8 & - & - & 10 & 0.3 \\
\hline $\begin{array}{l}\text { Not determined } \\
p=0.058\end{array}$ & 1013 & 39.5 & 131 & 35.8 & 99 & 36.2 & 1243 & 38.8 \\
\hline
\end{tabular}

Methods

We identified all women registered on the New Zealand Cancer Registry (NZCR) with a primary diagnosis of corpus uteri and uterus part unspecified cancer [International Classification of Diseases (ICD) ICD-10 codes C54-C55, ICD-9 code 182] during the period
1 January 1997 to 31 December 2006. The data extracted from the NZCR included tumour grade and stage, histology subtypes and basic demographic information, including age at diagnosis, ethnicity, and age at death.

Uterine cancer tumour grade was characterised according to the NZCR criteria, using four categories: well 
Table 2 Age-specific incidence rates per 100000 of uterine cancer, by ethnicity, 1997-2006

\begin{tabular}{|c|c|c|c|c|c|c|c|c|}
\hline \multirow[b]{2}{*}{ Age group (years) } & \multicolumn{2}{|l|}{$n M n P$} & \multicolumn{2}{|l|}{ Māori } & \multicolumn{2}{|l|}{ Pacific } & \multicolumn{2}{|c|}{ All women } \\
\hline & Cases $(n)$ & Rate & Cases $(n)$ & Rate & Cases $(n)$ & Rate & Cases $(n)$ & Rate \\
\hline $15-19$ & 1 & 0.1 & - & - & 1 & 0.8 & 2 & 0.1 \\
\hline $20-24$ & 2 & 0.2 & - & - & - & - & 2 & 0.1 \\
\hline $25-29$ & 2 & 0.2 & 2 & 0.8 & 2 & 2.0 & 6 & 0.4 \\
\hline $30-34$ & 17 & 1.4 & 8 & 3.4 & 9 & 9.3 & 34 & 2.2 \\
\hline $35-39$ & 28 & 2.2 & 9 & 4.1 & 14 & 15.9 & 51 & 3.2 \\
\hline $40-44$ & 62 & 4.9 & 31 & 16.2 & 21 & 28.8 & 114 & 7.5 \\
\hline $45-49$ & 130 & 11.1 & 46 & 29.9 & 24 & 41.4 & 200 & 14.5 \\
\hline $50-54$ & 249 & 23.7 & 66 & 58.2 & 32 & 70.6 & 347 & 28.7 \\
\hline $55-59$ & 395 & 44.5 & 61 & 70.0 & 32 & 91.4 & 488 & 48.3 \\
\hline $60-64$ & 362 & 49.0 & 46 & 69.3 & 48 & 180.1 & 456 & 54.9 \\
\hline $65-69$ & 348 & 54.1 & 47 & 95.8 & 42 & 202.3 & 437 & 61.3 \\
\hline $70-74$ & 332 & 56.8 & 21 & 66.0 & 21 & 145.5 & 374 & 59.3 \\
\hline $75-79$ & 252 & 49.2 & 17 & 89.4 & 17 & 188.3 & 286 & 52.9 \\
\hline $80-84$ & 226 & 58.9 & 7 & 71.6 & 7 & 142.3 & 240 & 60.2 \\
\hline $85+$ & 158 & 46.3 & 5 & 86.5 & 3 & 101.3 & 166 & 47.5 \\
\hline Total/ASR & 2564 & 9.9 & 366 & 17.7 & 273 & 32.4 & 3,203 & 11.4 \\
\hline
\end{tabular}

The crude incidence rate for all women was 19.9 per 100000.

ASR, age-standardised rates standardised to Segi's'13 (1960) population weights; nMnP, non-Māori non-Pacific women.

differentiated, moderately differentiated, poorly differentiated, and undetermined or unknown grades. The New Zealand Health Information Service (NZHIS) used the numeric extent of disease (stage) codes, assigned by cancer registrars, which were applied to registrations up to and including $1998 .{ }^{10}$ From 1999, the extent of disease coding was standardised using the Surveillance, Epidemiology and End Results (SEER) Guide to Summary Staging. ${ }^{11}$ Thus combining the SEER guide and the numeric code, we categorised tumour stage into four categories: local, regional, distant and not known.

For descriptive analyses, age at diagnosis was divided into 155 -year age bands from 15-19 years to 85 years and older. For logistic regression analyses, age was included as a continuous variable.

Ethnicity was classified using the standard New Zealand prioritisation system which gives the highest priority to Māori ethnicity (i.e. women who reported being Māori and also being in one or more other ethnic groups were classified as Māori) followed by Pacific ethnicity. Ethnicity was then classified into three categories: Māori, Pacific (i.e. Samoan, Cook Island Māori, Tongan, Niuean, Tokelauan, Fijian, other Pacific Island not listed and not further defined) and non-Māori nonPacific women (the majority of whom are European in origin, but this group also includes Asian, Middle Eastern, Latin American/Hispanic and African women, as well as those for whom ethnicity was not stated). ${ }^{10}$

To measure socioeconomic status, we converted domicile codes provided by the NZHIS to the New Zealand Deprivation Index 2006 (NZDep2006) as a standardised measure of socioeconomic deprivation. Based on the 2006 New Zealand Census, the index combines nine census variables, and provides a summary deprivation score from 1 to 10 for small area units, which contain a median of 90 people. A score of 1 is allocated to the least deprived $10 \%$ of areas and 10 is allocated to the most deprived $10 \%$ of areas. ${ }^{12}$ For our analyses, deciles were grouped into quintiles: $1-2$ (least deprived); 3-4; 5-6; 7-8; 9-10 (most deprived).

\section{Analysis}

The analyses were performed with the Stata (version 8.2) statistical package (StataCorp, College Station, TX, USA). Chi-square $\left(\chi^{2}\right)$ tests were used to examine ethnic differences for descriptive characteristics. Logistic regression was used to estimate odds ratios (ORs) and 95\% confidence intervals (CIs) for the associations between ethnicity, socioeconomic deprivation and tumour features (stage and grade). The level of statistical significance was accepted at the conventional level of $p \leq 0.05$. Two logistic models were run for each dependent variable (grade and stage of tumour); the first model involved estimation of the age-adjusted OR for ethnicity (or deprivation), and the second model included adjustment for deprivation (or ethnicity).

Uterine cancer incidence (or mortality) was estimated as the number of new registered uterine cases (or uterine cancer deaths) (aged 15+ years) during 1997-2006 per 100000 person-years. For the denominator, we obtained census population estimates from 1997 to 2006 for each ethnic group: Pacific, Māori and non-Māori non-Pacific peoples. Age standardisation of incidence and mortality rates was conducted using Segi's 1960 World Population. ${ }^{13}$ Mortality to incidence $(\mathrm{M}: \mathrm{I})$ ratios were calculated for each ethnic group, as a measure of prognosis.

Missing data $(n=14)$ were excluded from the analyses, where domicile codes could not be matched to the 
Table 3 Age-specific mortality rates per 100000 of uterine cancer, by ethnicity, 1997-2006

\begin{tabular}{|c|c|c|c|c|c|c|c|c|}
\hline \multirow[b]{2}{*}{ Age group (years) } & \multicolumn{2}{|l|}{$\mathrm{nMnP}$} & \multicolumn{2}{|l|}{ Māori } & \multicolumn{2}{|l|}{ Pacific } & \multicolumn{2}{|l|}{ All women } \\
\hline & Cases $(n)$ & Rate & Cases $(n)$ & Rate & Cases $(n)$ & Rate & Cases $(n)$ & Rate \\
\hline $15-19$ & 1 & 0.1 & - & - & - & - & 1 & 0.1 \\
\hline $20-24$ & - & - & - & - & - & - & - & - \\
\hline $25-29$ & - & - & - & - & - & - & - & - \\
\hline $30-34$ & 1 & 0.1 & - & - & - & - & 1 & 0.1 \\
\hline $35-39$ & 2 & 0.2 & 2 & 0.9 & 2 & 2.3 & 6 & 0.4 \\
\hline $40-44$ & 4 & 0.3 & 5 & 2.6 & 3 & 4.1 & 12 & 0.8 \\
\hline $45-49$ & 16 & 1.4 & 15 & 9.8 & 4 & 6.9 & 35 & 2.5 \\
\hline $50-54$ & 36 & 3.4 & 12 & 10.6 & 5 & 11.0 & 53 & 4.4 \\
\hline $55-59$ & 60 & 6.7 & 17 & 19.5 & 11 & 31.4 & 88 & 8.7 \\
\hline $60-64$ & 75 & 10.1 & 21 & 31.6 & 12 & 45.0 & 108 & 13.0 \\
\hline $65-69$ & 100 & 15.5 & 17 & 34.7 & 22 & 105.9 & 139 & 19.5 \\
\hline $70-74$ & 112 & 19.6 & 19 & 59.7 & 11 & 76.2 & 142 & 22.5 \\
\hline $75-79$ & 143 & 27.9 & 14 & 73.6 & 17 & 188.3 & 174 & 32.2 \\
\hline $80-84$ & 139 & 36.2 & 9 & 92.0 & 4 & 81.3 & 152 & 38.1 \\
\hline $85+$ & 210 & 61.6 & 7 & 121.1 & 4 & 135.1 & 221 & 63.2 \\
\hline Total/ASR & 899 & 2.6 & 138 & 7.4 & 95 & 12.1 & 1132 & 3.2 \\
\hline
\end{tabular}

NZDep2006 index. Information on other potentially important known risk factors, such as non-use of the oral contraceptive pill, type and duration of hormone replacement therapy, age at menarche, co-existing medical conditions (e.g. diabetes and hypertension) and other important reproductive factors were not available from this database. Smoking history is recorded on the NZCR database, but more than $70 \%$ of the data was incomplete, and thus it was not included in the analyses.

\section{Results}

There were 3203 women who had registered on the NZCR with a primary diagnosis of uterine cancer from 1997 to 2006. This comprised 2564 non-Māori non-Pacific, 366 Māori, and 273 Pacific women. The unadjusted average age at first diagnosis was 63.7 years (56.9 years for Māori women, 58.1 years for Pacific women, compared to 65.3 years for nonMāori non-Pacific women) $(p<0.001)$. Table 1 shows the distributions of clinical and demographic characteristics, by ethnicity. In this study, a large proportion of New Zealand women diagnosed with uterine cancer were living in lower socioeconomic deprivation areas (NZDep2006 7-0 decile groups). Across all ethnic groups, the majority of women were diagnosed with 'regional' stage of disease, although Pacific women had the highest chance of being diagnosed with distant metastases, as well as not having sufficient diagnostic tests to allow stage to be classified. For tumour grade, 38\% of all women had tumours coded as 'undetermined', 'unknown', 'not supplied' or 'not applicable' and therefore they do not have a histological grade assigned. However, among those who did have histology performed, many were well-differentiated tumours. Endometrioid adenocarcinoma was the most commonly recorded histological subtype of uterine cancer for all women (Table 1).

In every age group, Pacific women (followed by Māori) have higher incidence (Table 2) and mortality rates for uterine cancer (Table 3), compared to non-Māori non-Pacific women. Table 4 shows the M:I ratios using the age-standardised rates, estimated as a measure of prognosis. Māori and Pacific women have higher values of the M:I ratio than non-Māori non-Pacific women, which suggest Māori women with uterine cancer do not have a prognosis.

Age and ethnicity adjusted ORs showing the associations between socioeconomic position and tumour features are shown in Table 5. The age-adjusted analyses suggested that women from more deprived areas were less likely to present with well-differentiated tumours, but this effect was attenuated following adjustment for ethnicity. Women in the most deprived areas were more likely to present with an advanced stage of uterine cancer, and the association was attenuated after adjustment for ethnicity. The age and deprivation adjusted models (Table 6) showed that Māori and Pacific women are less likely to present with well-differentiated tumours, and that little of the effect appears to be due to confounding by deprivation. Pacific women were two and a half times more likely to present with 'advanced' stage uterine tumours, compared to non-Māori nonPacific women.

\section{Discussion}

Our study has shown that cancer of the uterus is a common gynaecological cancer affecting women in 
Table 4 Mortality to incidence ratio, by ethnicity

\begin{tabular}{|c|c|c|c|c|c|c|c|c|}
\hline & \multicolumn{2}{|c|}{$\mathrm{nMnP}$} & \multicolumn{2}{|c|}{ Māori } & \multicolumn{2}{|c|}{ Pacific } & \multicolumn{2}{|c|}{ All women } \\
\hline & I & $M$ & 1 & $M$ & $I$ & $M$ & I & $M$ \\
\hline & 2.6 & 9.9 & 17.7 & 7.4 & 32.4 & 12.1 & 11.43 & 3.2 \\
\hline M:I ratio & 0.26 & & 0.42 & & 0.37 & & 0.29 & \\
\hline
\end{tabular}

I, incidence; $\mathrm{M}$, mortality; nMnP, non-Māori non-Pacific women.

New Zealand in the older age group, and it is more common in Pacific and Māori (intermediary) women. Within this particular group of women, approximately half resided in the lowest deprived areas. Recent cancer analyses in New Zealand showed an increasing trend (by 17\%) of endometrial incidence rates among those aged $25+$ years old in the low-income group, compared to a $15 \%$ decrease in incidence in the high income group, from the early 1980 s to $2004 .{ }^{14}$ Our study further supports the notion of notable inequalities by socioeconomic status among women with uterine cancer. New Zealand women with uterine cancer have a reasonably good prognosis $(\mathrm{M}: \mathrm{I}=0.29)$ when diagnosed. This is probably because more than $70 \%$ of tumours were detected at an early stage, when treatment can be more effective. The M:I ratios appear to be elevated for Māori and Pacific women; however, this may be reflective of later diagnosis in Māori and Pacific women.

Recently, it had been reported that there was no strong evidence of an increasing trend in uterine cancer rates in all New Zealand women, except among Pacific women where rates have increased to $69 \%$ (95\% CI 55.1-84.2) in 2001-2004 from 89\% (95\% CI 17.3-57.6) in 1981-1986. ${ }^{14}$ Our study also demonstrates higher age-standardised rates of uterine cancer among Pacific women from 1997 to 2006, particularly among women in menopause. ${ }^{15}$ However, few studies have examined ethnic differences in uterine cancer among groups in New Zealand or internationally. Our study confirms that there is substantial variation in the incidence of uterine cancer by ethnicity, with the highest incidence in Pacific and Māori women. For Pacific women, the rate was almost double that of nonMāori non-Pacific women. This differs from results reported elsewhere from studies that have specifically looked at ethnic differences in uterine cancer. ${ }^{16}$ A USA study found that White American women had the highest age-adjusted rate (116.1 per 100000 women) compared to African-American (87.8 per 100000 women), native Hawaiian (106.7 per 100000 women), Japanese-American (71.3 per 100000 women) and Latina women (73.4 per 100000 women). ${ }^{16}$ The same authors also reported that among African-American women the incidence rate of advanced disease was $80 \%$ higher than that for White American women. ${ }^{16}$ Similarly, in the current study, there were significantly higher proportions of Pacific women who presented with advanced stage disease, which may explain in
Table 5 Association between deprivation level and tumour features: grade and stage

\begin{tabular}{lcccc}
\hline & $\mathbf{O R}^{*}$ & $\mathbf{9 5 \%} \mathbf{C l}$ & $\mathbf{O R}^{+}$ & $\mathbf{9 5 \%} \mathbf{C l}$ \\
\hline $\begin{array}{lllll}\text { Grade of tumour } \\
\quad\end{array}$ & & & & \\
$\quad$ NZDep 1 & 1.00 & & & \\
NZDep 2 & 0.90 & $0.64-1.26$ & 0.92 & $0.66-1.28$ \\
NZDep 3 & 0.87 & $0.63-1.19$ & 0.89 & $0.65-1.23$ \\
NZDep 4 & 0.85 & $0.63-1.15$ & 0.89 & $0.66-1.20$ \\
NZDep 5 & 0.78 & $0.60-1.06$ & 0.88 & $0.64-1.21$ \\
Stage of tumour & & & & \\
NZDep 1 & 1.00 & & & \\
NZDep 2 & 1.17 & $0.74-1.88$ & 1.14 & $0.71-1.82$ \\
NZDep 3 & 1.17 & $0.74-1.83$ & 1.10 & $0.70-1.72$ \\
NZDep 4 & 0.96 & $0.62-1.49$ & 0.89 & $0.58-1.39$ \\
NZDep 5 & 1.64 & $1.09-2.48$ & 1.32 & $0.86-1.97$ \\
\hline
\end{tabular}

${ }^{*}$ Adjusted for age.

${ }^{\dagger}$ Adjusted for age and ethnicity.

"Well-differentiated tumours vs moderately/poor tumours ( $n=1950)$.

§Distant/metastases vs other $(n=2879)$.

NZDep 1-5 is a standard measure of socioeconomic deprivation, where

NZDep 1 is the referent and is defined as 'least deprived' to NZDep 5,

which is the 'most deprived'.

$\mathrm{Cl}$, confidence interval; $\mathrm{OR}$, odds ratio.

part, their higher mortality, compared to non-Māori non-Pacific women.

There are several possible explanations for the higher rate of uterine cancer among Pacific women in New Zealand. First, it has been suggested that Pacific women of this generation are not common users of the combined oral contraceptive pill, which is known to be a protective factor for uterine cancer. ${ }^{5}$ The reasons for this include the lack of adequate education on contraceptive use, and the fact that Pacific (and Māori) women are significantly less likely to use contraceptives in general. ${ }^{17}$ However, the low use of oral contraception in Pacific women is more likely to be offset by the hormonal effects of frequent pregnancies. ${ }^{18}$ Furthermore, previous studies have found that hormone contraceptive users are typically characterised as being of White/European ethnicity, having a higher level of education, being thinner and older. ${ }^{19}$ The availability of ethnic-specific data on factors such as oral contraceptive use, type and duration over a life-course would provide necessary knowledge for preventive medicine, not just in this area, but for reproductive cancers in general. Second, higher rates of uterine cancer among Pacific women could be explained, in part, by obesity, which has been consistently reported as an independent risk factor for uterine cancer. ${ }^{15} 20$ ${ }^{21}$ The biological mechanism underlying this relationship remains unclear; however, the potential explanation includes the role of obesity in the production of peripheral oestrogens, primarily through the conversion of androstenedione to estrone by aromatase in adipose tissue, increasing the risk of uterine hyperplasia and hence cancer of the uterus. ${ }^{2223}$ Furthermore, the link between obesity and polycystic ovarian syndrome 
Table 6 Association between ethnicity and tumour features: grade and stage

\begin{tabular}{|c|c|c|c|c|}
\hline & OR* & $95 \% \mathrm{Cl}$ & $\mathrm{OR}^{\dagger}$ & $95 \% \mathrm{Cl}$ \\
\hline \multicolumn{5}{|c|}{ Tumour grade\# } \\
\hline $\mathrm{nMnP}$ & 1.00 & & & \\
\hline Māori & 0.69 & $0.52-0.92$ & 0.71 & $0.53-0.96$ \\
\hline Pacific & 0.72 & $0.52-0.99$ & 0.76 & $0.54-1.06$ \\
\hline \multicolumn{5}{|c|}{ Tumour stage } \\
\hline $\mathrm{nMnP}$ & 1.00 & & & \\
\hline Māori & 1.45 & $0.99-2.12$ & 1.32 & $0.88-1.97$ \\
\hline Pacific & 2.62 & $1.81-3.79$ & 2.45 & $1.66-3.60$ \\
\hline \multicolumn{5}{|c|}{$\begin{array}{l}{ }^{*} \text { Adjusted for age. } \\
{ }^{\circ} \text { Adjusted for age and NZDep06 quintile. } \\
\text { \#Well-differentiated tumours vs moderately/poor differentiated tumours } \\
(n=1950) \text {. } \\
\text { §Distant vs other stage ( } n=2891) \text {. } \\
\text { Cl, confidence interval; nMnP, non-Māori non-Pacific women; OR, odds } \\
\text { ratio. }\end{array}$} \\
\hline
\end{tabular}

(PCOS), leading to the production of high levels of unopposed estradiol, has also been reported as a significant risk factor in uterine hyperplasia. However, the prevalence of PCOS among Pacific women has not been adequately examined. In Auckland, New Zealand a cross-sectional study reported little or no symptoms of PCOS among Pacific Island women compared to other ethnic groups (European, Maori, Indian and Asian), yet the majority of Pacific women in that study were morbidly obese and had the highest rates of insulin resistance and lipid abnormalities. ${ }^{24}$ Moreover, prior research has suggested that having a high body mass index (BMI) from the mid-teens and for a period of 30 years thereafter, and closer to the time of diagnosis, is a strong risk predictor of uterine cancer. Obtaining accurate data on obesity and BMI information requires a life-course epidemiological approach that could provide important information specific to at-risk ethnic groups. ${ }^{25} 26$

The higher rates of uterine cancer among Pacific and Māori women are puzzling because they reportedly have higher fertility rates (median age 27.7 years and 26.0 years, respectively) compared to all women (32.0 years) and they tend to have larger families. ${ }^{18}$

There is clear evidence from previous research that pregnancy (ever vs never) ${ }^{27}$ has a protective effect on uterine cancer, which increases with increasing parity. ${ }^{28}$ However, this protective effect is either not present in Pacific and Māori women, or is outweighed by other uterine cancer risk factors. Pacific and Māori women are diagnosed with uterine cancer at a younger age, which could be an indicator of familial predisposition. ${ }^{29}$ Some studies have reported differences in the genes responsible for hormonally responsive cancers, such as prostate and breast cancer, but these studies need to be extended to further large and well-characterised association studies. ${ }^{30}$ Given the different rates of diagnosis of uterine cancer, it may be important to consider whether specific genes are involved in hormone function and metabolism, and may act as predisposition genes for specific ethnic populations.

We found that women living in the most deprived areas were less likely to have well-differentiated tumours, albeit with more advanced stage of the disease, suggesting a socioeconomic gradient in risk of women living in more deprived areas, and this raises questions about access to diagnostic services for these groups of women. Furthermore, Māori and Pacific women were also less likely to have well-differentiated tumours compared to non-Māori non-Pacific women. Although this association remained significant for Māori, for Pacific women the association reduced when adjusted for deprivation. Pacific women were significantly more likely to have an advanced stage of disease at initial diagnosis (age and deprivation adjusted) compared to non-Māori non-Pacific women. Apart from the usual risk factors (e.g. obesity, poor access to health services, co-morbid conditions) it is possible that Pacific women are presenting with a more aggressive histological subtype. This hypothesis appears to be valid for African-American women who had a much higher risk of developing cancer with more aggressive histology in a USA study. ${ }^{5}$

\section{Limitations of the study}

The limitations of this study are: (1) the lack of specific histological information [e.g. International Federation of Gynecology and Obstetrics (FIGO) codes]; (2) the lack of information on other potential risk factors (e.g. oral contraceptive use, smoking history, parity vs nulliparity) that are not recorded in the NZCR; (3) the precision of our ethnic-specific rate estimates may be hampered by the small number of Pacific and Māori cases; ${ }^{31}$ although the NZCR endeavours to differentiate between New Zealand resident and non-resident registrants, there is still the potential that some nonresident registrants have been included in our analyses, resulting in inflated incidence rates. Furthermore, some cancer registrations with unspecified ethnicity were included in the non-Māori non-Pacific group, which would have reduced the observed differences between ethnic groups; and (4) the final limitation relates to a considerable proportion of women in our sample with missing 'tumour graded' data. This could be due to errors in coding, or that histopathology samples were not taken, or that the different information sources (clinical, radiological, histological, autopsy or death certificate) providing cancer diagnosis were incomplete. The lack of complete information on tumour grade at diagnosis will affect the precision of the estimates produced by this study, introducing the possibility of selection bias.

\section{Conclusions}

This study has provided some useful findings comparing New Zealand women with uterine cancer, by ethnic group. We have shown that there are ethnic differences 
in uterine cancer incidence and mortality, and that the differences in stage and grading of tumours are not completely explained by age, ethnicity and deprivation. More information around the known and unknown risk factors of this disease would sufficiently complete the knowledge gaps, especially for high-risk ethnic groups. Our study highlights some significant differences in disease presentation, by ethnic groups. In light of the findings from our study, further investigations are necessary to examine other explanatory or causal factors of uterine cancer. These factors could include occupational exposures, domestic and external environmental factors, diet composition, lifestyle and possibly genetics.

Acknowledgement Dr R T Firestone was funded by a Pacific Postdoctoral Fellowship from the Health Research Council of New Zealand.

\section{Competing interests None.}

Provenance and peer review Not commissioned; externally peer reviewed.

\section{References}

1 Curado MP, Edwards B, Shin HR, et al. Cancer Incidence in Five Continents (Vol. IX, IARC Scientific Publication No. 160). Lyon, France: International Agency for Research on Cancer (IARC), 2007.

2 Parkin DM, Whelan SL, Ferlay J, et al. Cancer Incidence in Five Continents. (Vol. VIII, IARC Scientific Publication No. 155). Lyon, France: International Agency for Research on Cancer (IARC), 2002.

3 Ministry of Health. Cancer: New Registrations and Deaths 2005: Revised Edition. New Zealand, Wellington: Ministry of Health, 2009.

4 Ministry of Health. Cancer in New Zealand - Trends and Projections. Wellington, New Zealand: Ministry of Health, 2000.

5 Purdie DM, Green AC. Epidemiology of endometrial cancer. Best Pract Res Clin Obstet Gynaecol 2001;15:341-354.

6 Jensen KE, Hannibal CG, Nielsen A, et al. Social inequality and incidence of and survival from cancer of the female genital organs in a population-based study in Denmark, 1994-2003. Eur J Cancer 2008;44:2003-2017.

7 Foliaki S, Jeffreys M, Wright C, et al. Cancer in Pacific people in New Zealand: a descriptive study. Pac Health Dialog 2004;11:94-100.

8 Robinson B, Purdie G, Cormack D. Unequal Impact: Maori and non-Maori Cancer Statistics 1996-2001. Wellington, New Zealand: Ministry of Health, 2006.

9 Tukuitonga CF, Solomon N, Stewart A. Incidence of cancer among Pacific Island people in New Zealand. N Z Med J 1992;105:463-466.

10 New Zealand Health Information Service. New Zealand Cancer Registry Data Dictionary. Wellington, New Zealand: New Zealand Health Information Service, 2004.

11 National Cancer Institute. Surveillance, Epidemiology and End Results (SEER) Extent of Disease 1988 Codes and Coding Instructions. US Department of Health and Human Services. Cancer Statistics Branch Surveillance Program. Bethesda, MD: National Cancer Institute, 1989.

12 White P, Gunston J, Salmond C, et al. Atlas of Socioeconomic Deprivation in New Zealand NZDep2006. Wellington, New Zealand: Ministry of Health, 2008.
13 Segi M. Cancer Mortality for Selected Sites in 24 Countries (1950-1957). Sendai, Japan: Tohoku University of Medicine, 1960.

14 Blakely T, Shaw C, Atkinson J, et al. Cancer Trends: Trends in Incidence by Ethnic and Socio-economic Group, New Zealand 1981-2004. Wellington, New Zealand: University of Otago and the Ministry of Health, 2010.

15 Allen NE, Key TJ, Dossus L, et al. Endogenous sex hormones and endometrial cancer risk in women in the European Prospective Investigation into Cancer and Nutrition (EPIC). Endocr Relat Cancer 2008;15:485-497.

16 Setiawan VW, Pike MC, Kolonel LN, et al. Racial/ethnic differences in endometrial cancer risk: the multiethnic cohort study. Am J Epidemiol 2007;165:262-270.

17 Young LK, Farquhar CM, McCowan LM, et al. The contraceptive practices of women seeking termination of pregnancy in an Auckland clinic. N Z Med J 1994;107:189-192.

18 New Zealand Health Information Service. Report on Maternity: Maternal and Newborn Information 2004. Wellington, New Zealand: Ministry of Health, 2007.

19 Egeland GM, Matthews KA, Kuller LH, et al. Characteristics of noncontraceptive hormone users. Prev Med 1988;17: 403-411.

20 Bergström A, Pisani P, Tenet V, et al. Overweight as an avoidable cause of cancer in Europe. Int J Cancer 2001;91:421-430.

21 Bjørge T, Engeland A, Tretli S, et al. Body size in relation to cancer of the uterine corpus in 1 million Norwegian women. Int J Cancer 2007;120:378-383.

22 Calle EE, Rodriguez C, Walker-Thurmond K, et al. Overweight, obesity, and mortality from cancer in a prospectively studied cohort of U.S. adults. N Engl J Med 2003;348:1625-1638.

23 Persson I, Adami, H-O. Endometrial cancer. In: Adami H-O, Hunter D, Trichopoulos D (eds), Textbook of Cancer Epidemiology. New York, NY: Oxford University Press, 2002.

24 Williamson K, Gunn AJ, Johnson N, et al. The impact of ethnicity on the presentation of polycystic ovarian syndrome. Aust N Z J Obstet Gynaecol 2001;41:202-206.

25 Olson SH, Trevisan M, Marshall JR, et al. Body mass index, weight gain, and risk of endometrial cancer. Nutr Cancer 1995;23:141-149.

26 Swanson CA, Potischman N, Wilbanks GD, et al. Relation of endometrial cancer risk to past and contemporary body size and body fat distribution. Cancer Epidemiol Biomarkers Prev 1993;2:321-327.

27 Brinton LA, Berman ML, Mortel R, et al. Reproductive, menstrual, and medical risk factors for endometrial cancer: results from a case-control study. Am J Obstet Gynecol 1992;167:1317-1325.

28 Wang-Hong X, Yong-Bing X, Zhi-Xian R, et al. Menstrual and reproductive factors and endometrial cancer risk: results from a population-based case-control study in urban Shanghai. Indian J Cancer 2004;108:613-619.

29 Devivo I, Persson I, Adami H. Endometrial cancer. In: Adami H, Hunter D (eds), A Textbook of Cancer Epidemiology (2nd edn). New York, NY: Oxford University Press, 2008.

30 Kolonel LN, Altshuler D, Henderson BE. The multiethnic cohort study: exploring genes, lifestyle and cancer risk. Nat Rev Cancer 2004;4:519-527.

31 Kelsey JL, LiVolsi VA, Holford TR, et al. A case-control study of cancer of the endometrium. Am J Epidemiol 1982;116:333-342. 